\title{
Tabularia
}

TABULARIA Sources écrites des mondes normands médiévaux Les femmes et les actes | 2004

\section{De la Vicomtesse Emma et de son entourage}

Viscountess Emma and her circle

\section{Manon Six}

\section{CpenEdition \\ Journals}

\section{Édition électronique}

URL : http://journals.openedition.org/tabularia/1630

DOI : $10.4000 /$ tabularia. 1630

ISSN : $1630-7364$

Éditeur :

CRAHAM - Centre Michel de Boüard, Presses universitaires de Caen

\section{Référence électronique}

Manon Six, «De la Vicomtesse Emma et de son entourage », Tabularia [En ligne], Les femmes et les actes, mis en ligne le 13 juillet 2004, consulté le 01 mai 2019. URL : http://journals.openedition.org/ tabularia/1630 ; DOI : 10.4000/tabularia.1630 


\title{
De la Vicomtesse Emma et de son entourage Viscountess Emma and her circle
}

\author{
Manon SIX \\ Étudiante, Université de Paris VII ${ }^{1}$ \\ sixmanon@hotmail.com
}

Résumé:

Mystérieuse carrière que celle d'Emma la Vicomtesse dans la seconde moitié du XII ${ }^{e}$ siècle... Entre la Normandie, l'Angleterre et la région parisienne, elle prend à ferme les revenus ducaux, pratique le commerce et apparaît dans des nécrologes d'établissements ecclésiastiques. Le roi Henri II, imité par de grands personnages de la cour, lui apporte son soutien, tandis qu'elle fréquente les élites bourgeoises, les marchands et les prêteurs d'argent qui accompagnent l'émergence de la commune de Rouen. Alors que deux de ses fils prennent une part active au gouvernement communal, on ignore tout de l'origine sociale de la Vicomtesse, qui n'est jamais associée à un mari, mais accède à des fonctions importantes et assume des responsabilités rares pour une femme seule à cette époque.

Mots-clés : femmes, pouvoir, vicomté, Rouen, commerce, bourgeoisie, commune, famille.

\begin{abstract}
:
The career of Viscountess Emma, in the second half of the $12^{\text {th }}$ century, was indeed a mysterious one... Between Normandy, England and the Paris region, she farmed out the income of the $d u$ chy, engaged in trade and appeared in the obituary registers of ecclesiastical institutions. King Henry II, his example followed by high-ranking members of his court, lent her his support, while she frequented the citizen elite, the merchants and the money-lenders involved in the emergence of the Commune of Rouen. Two of her sons played an active part in the governing of the town, but nothing is known of the social origins of the Viscountess, who was never considered in association with a husband, yet rose to positions of importance and took on responsibilities rarely assumed at that time by a woman acting in her own right.
\end{abstract}

Keywords: women, power, vicomté, Rouen, trade, bourgeoisie, commune, family.

La médiocrité des sources de l'histoire des femmes, encore au XII ${ }^{e}$ siècle, constitue un obstacle réel à la réalisation de portraits des figures féminines de cette époque. Alors que les femmes de l'aristocratie et les religieuses demeurent les mieux étudiées,

1. Cet article reprend les thèmes abordés dans un mémoire de DEA, «Les bienfaiteurs rouennais de l'abbaye Saint-Ouen de Rouen pendant la période ducale, $\mathrm{XI}^{\mathrm{e}}-\mathrm{XII}{ }^{\mathrm{e}}$ siècles », préparé sous la direction de M. Mathieu Arnoux, Université Paris VII-Denis Diderot, 2002, dactyl., 180 p. Je remercie M. Pierre Bauduin, M. Mathieu Arnoux et $\mathrm{M}^{\text {me }}$ Véronique Gazeau pour les remarques et les pistes de recherches qui ont enrichi cet article. 
très peu de renseignements nous sont parvenus sur la vie des femmes à la ville; pourtant, il y naissait à cette époque de nouveaux types d'hommes et de femmes et par conséquent, de nouveaux types de rapports sociaux, dans la perspective d'une certaine autonomie économique qui nous échappe encore. Les femmes sont généralement étudiées à travers les stratégies familiales d'alliance et les mariages, les héritages et les droits de succession, les dots, les douaires, mais rarement à travers leurs actions elles-mêmes. Les reines sont perçues comme auxiliaires du gouvernement en temps de crise, assurant la régence pendant la minorité des enfants, ou comme initiatrices de fondations religieuses. Leur rôle pouvait toutefois consister en une force de persuasion non négligeable. Elles sont les seules au début du XII ${ }^{e}$ siècle à posséder leurs propres sceaux, suivies à la fin du siècle par des femmes d'une noblesse plus commune, bénéficiant à leur tour d'un moyen d'expression de leur autorité dans leurs actes. Symbole d'une certaine reconnaissance de leur statut, l'utilisation de ces sceaux ne correspondait néanmoins qu'à un usage strictement personnel, sans dimension publique véritable ${ }^{2}$. Orderic Vital décrit les conditions des femmes de haute naissance avant le milieu du XII ${ }^{\mathrm{e}}$ siècle ${ }^{3}$ : les figures féminines d'envergure, éclipsant parfois leur mari, sont rares. De toute évidence, la femme accédait à plus d'autonomie et plus de liberté légale, à partir du moment où elle devenait veuve ${ }^{4}$. La figure d'Emma la Vicomtesse dans la seconde moitié du XII e siècle illustre toutefois le rôle que pouvait jouer une femme en matière de pouvoir et de politique. Mais comment cette ascension a-t-elle pu se faire, dans une société où la chance qu'avait une femme d'accéder au pouvoir ne dépendait que du mariage qu'elle contractait?

\section{Des éléments déjà connus}

La Vicomtesse Emma nous est principalement connue par la notice que Léopold Delisle lui a consacrée dans son Introduction aux actes de Henri II Plantagenêt ${ }^{5}$ : en plus d'une fille également appelée Emma, la Vicomtesse laissa au moins quatre fils, Hugues, Geoffroi, Henri et Guillaume dont deux, Hugues et Geoffroi, occupaient vraisemblablement une place notable dans la bourgeoisie rouennaise, figurant dans de nombreux actes souscrits par les maires de Rouen, à la fin du XII et au début

2. THOMPSON, Kathleen, «Matilda, countess of Perche (1171-1210): the expression of authority in name, style, and seal», Tabularia (http://www.unicaen.fr/mrsh/crahm/revue/tabularia/thompson.html et Tabularia «Études», n³, 2003, p. 69-88).

3. Chibnall, Marjorie, «Women in Orderic Vitalis», The Haskins Society Journal, vol. 2, 1990, p. 105121.

4. JoHNS, Susan, "The Wives and Widows of the Earls of Chester, 1100-1252: the Charter Evidence ", The Haskins Society Journal, vol. 7, 1995, p. 117-152. Également: JoHNS, Susan, Noblewomen, aristocracy and power in the twelfth-century Anglo-Norman realm, Manchester et New York, Manchester University Press, 2003, XII + 276 p.

5. Delisle, Léopold et BERGER, Élie, Recueil des actes de Henri II Plantagenêt, Recueil des Historiens de la France, Chartes et Diplômes, 1909, Introduction, p. 214-218 [ci-après, DELISLE et BERGER, Recueil...]. 
du XIII ${ }^{\mathrm{e}}$ siècle. Hugues jura la reddition de la ville en 1204 ; Geoffroi était notamment possesseur d'une terre à Franqueville près de Boos $^{6}$ et il donna aux lépreux de Rouen (très certainement ceux du Mont-aux-Malades) un revenu de 10 sous, dont une partie sur une terre tenue par Clarembaud le Roux, membre d'un grand lignage proche de la commune de Rouen ${ }^{7}$.

L. Delisle recense les auteurs des chartes dans lesquelles figure les deux frères. Geoffroi apparaît dans les chartes de:

- Guillaume, fils de Gocelin, du temps du maire Luc du Donjon (vers 1187$1200)^{8}$;

- Roger, archevêque d'York, à l'époque du maire Barthélémy Fergant (vers 1171-1190);

- Raoul, chancelier du roi d'Angleterre (maire : Barthélémy Fergant) ${ }^{9}$;

- Robert, comte de Leicester (maire: Luc du Donjon) ${ }^{10}$;

- Robert, prieur du Mont-aux-Malades (maire: Raoul de Cotevrard, vers 11931201);

- Robert le Juif (maire : Mathieu le Gros, vers 1195-1200);

- Silvestre de Foro (maire: Mathieu le Gros) ${ }^{11}$.

Hugues, fils de la Vicomtesse figure lui dans les chartes de:

- Raoul, chancelier du roi d'Angleterre, du temps de Barthélémy Fergant ${ }^{12}$;

- Barthélémy, Mathieu et Roger, fils de Barthélémy Bataille (maire: Raoul de Cailly, vers 1198);

- Guillaume, fils de Gocelin (maire : Luc du Donjon) ${ }^{13}$;

- Robert, comte de Leicester (maire: Luc du Donjon) ${ }^{14}$;

- Robert le Juif (maire : Mathieu le Gros).

L. Delisle précise également que les deux frères sont appelés Gaufridus vicecomes et Hugo, frater suus dans une charte de Roger de Warwick, chanoine de Rouen, rédigée en présence du maire Mathieu le Gros $^{15}$.

6. Franqueville: paroisse Saint-Pierre de Franqueville, Seine-Maritime, cant. de Boos; Boos, SeineMaritime, ch.-l. de cant., arrond. de Rouen.

7. Cartulaire de la Cathédrale de Rouen, Rouen, Arch. dép. Seine-Maritime, G 4365 et fonds de Saint-Ouen, 14H 916.

8. Paris, Arch. nat., S 5199, $\mathrm{n}^{\circ} 55$

9. Cartulaire de la cathédrale de Rouen, Rouen, Bibl. mun., ms Y44, n 84, fol. 68v.

10. Paris, Arch. nat., S 5199, $\mathrm{n}^{\circ} 56$.

11. Paris, Arch. nat., $\mathrm{S} 4899, \mathrm{n}^{\circ} 11$.

12. Cartulaire de la cathédrale de Rouen, Rouen, Bibl. mun., ms Y44, $\mathrm{n}^{\circ} 84$.

13. Paris, Arch. nat., S 5199, $\mathrm{n}^{\circ} 55$.

14. Paris, Arch. nat., S 5199, $\mathrm{n}^{\circ} 56$

15. Delisle et BERGER, Recueil..., Introduction, p. 214-218. Il est indiqué que toutes les chartes sans référence étaient contenues en 1849 dans un dossier intitulé « divers anciens titres entre particuliers passés devant le maire de Rouen », aux Archives départementales de la Seine-Maritime. 
Les liens de la famille de la Vicomtesse avec le mouvement canonial apparaissent si l'on prend en compte le quatrième fils d'Emma, Guillaume, clerc du diocèse de Lisieux, qui figure en compagnie de sa mère dans le nécrologe de Saint-Victor de Paris ${ }^{16}$. Hugues figurerait peut-être dans l'obituaire de l'abbaye Saint-Ouen de Rouen, au mois d'avril semble-t-il: "Hugo filius comitisse, xxvii sol. » ${ }^{17}$. Après les enfants, qualifiés uniquement par leur prénom et la mention « fils ou fille de la Vicomtesse », en supposant qu'Emma n'ait eu effectivement que cinq enfants, on perd la trace d'une possible descendance.

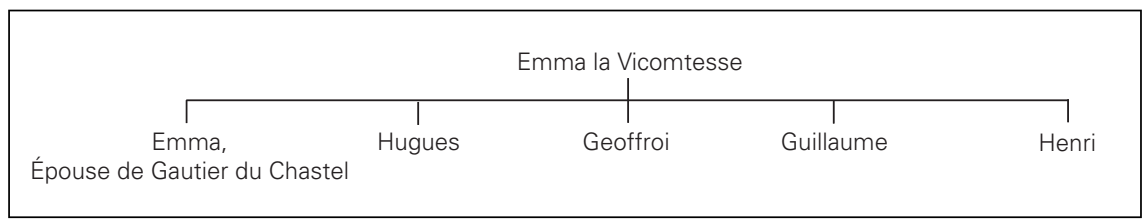

La descendance connue d'Emma la Vicomtesse.

Emma apparaît en 1158 dans des documents financiers sous ce nom de vicecomitissa Rothomago, alors qu'elle est en Angleterre: il est donc probable qu'elle ait été vicomtesse de Rouen auparavant. En Angleterre, elle reçut des sommes plus ou moins importantes, de 18 à 34 livres, sur ordre du roi, per breve Regis, sans que le motif de paiement ne fût jamais indiqué. En 1158, au plus tard, Emma fut chargée de la ferme de Southampton (Hantona), d'après le Pipe Roll de la $5^{\mathrm{e}}$ année du règne, car elle devait 237 livres et 4 sous de l'ancienne ferme et rendait compte pour la nouvelle. Elle succédait ainsi à Southampton à Guillaume Trentegerons, qui appartenait à une famille rouennaise ${ }^{18}$. De 1158 à 1163, les comptes indiquent que la Vicomtesse restait débitrice de sommes toujours plus importantes: de plus de 238 livres en 1158, elle resta redevable en 1163 de plus de 1423 livres ${ }^{19}$. En 1164, ce n'était plus la Vicomtesse qui rendait compte pour la ferme de Southampton. En 1165, le compte rendu par les mêmes fermiers que l'année précédente rappelle la situation de la Vicomtesse, toujours redevable à cette date de plus de 1423 livres, mais avec la mention qu'il ne lui serait plus réclamé cette somme plus longtemps ${ }^{20}$. Le roi semblait donc avoir décidé qu'il ne fallait plus tenir compte de sa faillite. Henri II devait avoir con-

16. Obituaire de Saint-Victor de Paris, éd. Auguste Molinier, Recueil des Historiens de la France, Obituaires de la province de Sens, t. I. Diocèse de Paris et de Sens, Paris, Imprimerie nationale, 1902, p. 555, 24 avril: «Item anniversarius Guillelmi, clerici Lexoviensis, et Vicecomitisse, matris ejus, de cujus beneficio habuimus viginti libras turonensis ad emendos redditus ".

17. Rouen, Bibl. mun., ms A459 (276), fol. 4, Missale Rothomagensis (XIII siècle), contenant un calendrier-obituaire de Saint-Ouen de Rouen.

18. Magni Rotuli Scaccarii Normannie sub Regibus Angliae, éd. Thomas Stapleton, Londres, Soc. Ant. Londinensis, 1840-1844, tome I, p. 80 et tome II, p. 306 [ci-après Magni Rotuli].

19. The Great Rolls of the Pipe for the [5th-34th] years of the reign of King Henry the second, ed. by The Pipe Rolls Society, Londres, 1884-1925: Pipe V, p. 50-51; Pipe VI, p. 22; Pipe VII, p. 58; Pipe VIII, p. 39; Pipe IX, p. 56.

20. Ibid., Pipe XII, p. 45. 
tracté des obligations à son égard, peut-être liées à un prêt reçu dans les années décisives de son avènement en Angleterre (English Pipe Rolls, 1160-1164). Emma serait alors revenue en Normandie, où elle aurait réussi à constituer une compagnie marchande ${ }^{21}$ et à devenir assez populaire, au point que plusieurs grands personnages de la cour ducale semblèrent se porter garants pour elle, afin qu'elle pût recouvrer la responsabilité de la ferme de la vicomté de Rouen. Mais l'entreprise fut à nouveau un échec, la Vicomtesse dut s'effacer en 1180 devant la commune de Rouen, laissant une dette d'un peu plus de 2214 livres, qui fut inscrite sur le rôle de l'Échiquier ${ }^{22}$. La somme est considérable quand on sait qu'en 1180, la ferme de la vicomté de Rouen et ses annexes rapportaient au duc près de 6000 livres. Les sommes engagées par les différents garants de la Vicomtesse y figuraient également: Richard Giffard avait promis 40 livres; Hugues de Cressy, 100 livres; Robert Marmion, 100 livres; Gérard de Canville, 100 livres ; Sehier de Quincy, un peu plus de 40 livres ; Robert de Brucourt, 30 livres $^{23}$. Ces personnalités étaient les témoins habituels des chartes de Henri II : ont-ils été contraints par le roi de verser ces sommes? Ou bien avaientils, eux aussi, eu recours aux services financiers de la Vicomtesse auparavant?

Par la suite, on sait qu'Emma continuait à être considérée comme débitrice du Trésor sous le règne de Richard Cour de Lion. En 1195, 30 ans après l'effacement de sa dette, on garde encore la trace d'un de ses garants, Guillaume Batles-bues, lui apportant une caution de 29 livres $^{24}$. Et deux mentions se rapportent à sa situation dans le rôle de 1198, où elle est simplement appelée "Emma vicecomitissa», unique fois où «Rothomago » n'est pas précisé ${ }^{25}$. Elle aurait versé à Mathieu le Gros, maire de Rouen, la somme dérisoire de 25 sous pour compenser son ahurissante dette : un peu plus de 2198 livres en $1198^{26}$. Après ce remboursement, elle restait donc redevable de 2197 livres 7 sous 6 deniers. Une charte du cartulaire de Saint-Lazare de Paris nous apprend par ailleurs qu'Emma la Vicomtesse était propriétaire de bateaux transportant du sel et des harengs jusqu'à Paris ${ }^{27}$.

21. D'après la mention contenue dans Magni Rotuli, t. II, p. 305: «Emma vicecomitissa de Rothomago et Rollandus cambitor, 40 marcas de debitis Gervasii de Hantonna», la Vicomtesse et Rolland le Changeur assumant ensemble la dette de Gervais de Southampton, une association marchande a pu être formée par les trois personnages. Un peu plus loin, un certain Ernaudus de Rothomago, autre membre présumé de cette compagnie, verse également 16 livres et 16 sous tournois pour la dette de Gervais de Southampton. D’après L. Delisle, Gervais de Southampton, qui ne vivait plus en 1196, avait fait partie d'une compagnie marchande, qui aurait eu à sa tête Nigellus de Havena auquel les agents financiers de Richard Cœur de Lion réclamaient une somme de 50 marcs inscrite sur le Pipe Roll de 1198 (Pipe IX, cité par Stapleton, Magni Rotuli, t. II, p. xxii).

22. Magni Rotuli, I, p. 78.

23. Magni Rotuli, I, p. cvi.

24. Magni Rotuli, I, p. 284.

25. Magni Rotuli, II, p. 395.

26. Magni Rotuli, op. cit., II, p. 305-306.

27. Delisle et Berger, Recueil..., Introduction, p. 364 ; «Cartulaire de Saint-Lazare de Paris » (11241254), Arch. nat., MM 210, fol. 41v; Magni Rotuli, II, p. xxii et 305. 
Entre 1180 (2214 livres environ) et 1198 (2198 livres environ), sa dette était remboursée très lentement. En raison de ces faibles acquittements, la dette de la Vicomtesse peut être perçue différemment, témoignant plus du soutien et de la protection accordés par le roi, que de la conséquence désastreuse d'une mauvaise gestion ${ }^{28}$. Les sommes ainsi perçues par elle provenant de personnages importants à la cour à cette époque, sur ordre du roi, sans motif exprimé, correspondraient plus volontiers à des marques de soutien, qu'à un effort solidaire pour l'acquittement de la dette.

Les informations s'épuisent ensuite. On sait qu'Emma avait fait construire à Saint-Wandrille ${ }^{29}$ une maison de pierre sur un terrain appartenant à l'abbaye. Un acte antérieur à 1178 la concernant, dans les sources de Saint-Wandrille, eut pour témoins deux membres de l'un des plus notables lignages bourgeois de Rouen, les Groignet ${ }^{30}$. De plus elle figurait dans l'obituaire de la cathédrale de Rouen, son obit se célébrant le 2 septembre : une considération évidente, car elle y était seule représentante de ce groupe social de marchands ${ }^{31}$.

\section{Emma la Vicomtesse et sa famille}

En l'absence avérée de patronyme pour les enfants de la Vicomtesse, la recherche de leur père et du mari d'Emma, en admettant qu'il n'y en ait eu qu'un seul, est ardue. Les arbres généalogiques esquissés plus bas supposent que les enfants de la Vicomtesse appartenaient à la même fratrie, mais toutes les combinaisons sont néanmoins possibles. Il est rare pour des enfants de conserver le nom, ou plutôt le titre, de leur mère seule. En partant du principe que le ou les pères disparurent très tôt, le seul prestige de la fonction de la mère expliquerait-il le choix de la dénomination des enfants? Il semble que toute paternité ait été complètement éclipsée. Emma la Vicomtesse n'est jamais associée dans ses activités à un mari, un père ou un frère et les enfants ne sont identifiés que grâce à la référence maternelle. Enfants illégitimes,

28. Je remercie aimablement M. Vincent Moss de m'avoir fait part de ces remarques. Il propose en effet de considérer davantage les grandes dettes contenues dans les rôles de l'Échiquier normand sous la forme d'actes de patronage, de soutiens accordés à une clientèle. Il suggère de voir plutôt la dette d'Emma la Vicomtesse comme une marque de protection royale, plutôt que comme une faillite administrative. Moss, Vincent, "Normandy and England in 1180: The Pipe Roll Evidence», in England and Normandy in the Middle Ages, David BATES et Anne CURRY (éd.), Londres et Rio Grande, 1994, p. 185-195; Moss, Vincent, «The Norman Fiscal Revolution», in Crises, Revolutions and Selfsustained Growth: Essays in European Fiscal History, 1130-1830, William M. Ormrod, Margaret Bonney, Richard Bonney (éd.), Oxford, 1999, p. 40-41; Moss, Vincent, «Reprise et innovations: les rôles normands et anglais de l'année 1194-1195 et la perte de la Normandie ", in La Normandie et l'Angleterre au Moyen Âge, Actes du colloque de Cerisy-la-Salle [4-7 oct. 2001], Pierre BouET et Véronique GAZEAU (dir.), Caen, Publications du CRAHM, 2003, p. 89-97.

29. Saint-Wandrille, Seine-Maritime, cant. Caudebec-en-Caux.

30. Cartulaire de Saint-Wandrille, Rouen, Arch. dép. Seine-Maritime, $16 \mathrm{H}$, fol. $117 \mathrm{v}, \mathrm{n}^{\circ} \mathrm{XV}$, acte de l'abbé Anfredus, vers 1165-1178.

31. Obituaire de la cathédrale de Rouen, Rouen, Arch. dép. Seine-Maritime, G 2094. 
hors mariage, issus de mésalliances entre la Vicomtesse et des hommes de lignages aristocratiques... les lacunes documentaires donnent libre cours à l'imagination.

Cependant, des suppositions peuvent voir le jour. Parmi ceux qui sont susceptibles d'avoir été l'époux d'Emma figure Guillaume Trentegerons. Au début du XIII ${ }^{e}$ siècle, deux groupes familiaux se distinguent clairement à Rouen : d'une part, une famille Trentegerons et d'autre part, le groupe de fils et filles de la Vicomtesse, dont Geoffroi «fils de la Vicomtesse». Or, le chef du lignage des Trentegerons, maire de Rouen en 1218, s'appelait précisément Geoffroi... On a vu que Guillaume Trentegerons prit à ferme la vicomté de Rouen vers 1150 ; il fut l'un des bailleurs de fonds qui aidèrent à financer les entreprises de Henri II contre le roi Étienne. Il reçut la ferme de Southampton en 1156, prise d'ailleurs au taux trop élevé de 300 livres sterling, qui le ruina. Quand il quitta ses fonctions en 1158, il laissait un déficit de 236 livres. Il figure pour la dernière fois sur le Pipe Roll de 1156 comme touchant 15 livres du sheriff du Sussex puis disparut.

Il fut très certainement à l'origine de la famille qui donna un maire à Rouen en 1218. Un certain Jean Trentegerons est cité dans les Grands Rôles de l'Échiquier de 1180 et 1198, pour des dettes mineures, la seconde fois à côté d'Emma la Vicomtesse ${ }^{32}$. La place qu'il occupait en 1198, aux côtés de la Vicomtesse, laisse penser qu'il aurait été associé à la ferme de la vicomté de Rouen. Gautier Trentegerons, frère de Guillaume, fut témoin, vers le milieu du XII ${ }^{e}$ siècle, d'une vente à Saint-Amand de Rouen. Avant 1169, Guillaume et Gautier prirent ensemble en charge une rente en poivre assise à Rouen et due à l'abbaye de Montebourg en Cotentin ${ }^{33}$. Geoffroi Trentegerons, lui, fit en 1202 des affaires avec la comtesse de Flandre en tant que «marchand de Rouen » puis devint maire de Rouen en 1218: la famille était donc solidement enracinée en Normandie et les affaires anglaises ne semblent pas l'avoir occupée longtemps.

Emma succéda donc à Guillaume dans la ferme de Southampton qu'elle garda jusqu'en 1163, au même prix de 300 livres. Il est intéressant de constater que le montant de la ferme resta le même, qu'il fût confié à un homme ou à une femme : à responsabilité égale, il n'y avait pas de disparité financière. On notera également que Guillaume Trentegerons lui-même ne sortit pas glorieux de ses fonctions de fermier de Southampton : la défaillance d'Emma n'était peut-être pas uniquement le signe d'une mauvaise gestion personnelle.

Mais une autre piste semble tout aussi intéressante pour retrouver un possible mari d'Emma. La Vicomtesse eut en effet une fille, Emma, qui vendit avec son mari Gautier du Chastel, ou de Châtillon, un peu avant 1182, à maître Gautier de Coutances, trésorier de Rouen, ses droits sur une maison ayant appartenu à Raoul Fils Étienne, par un acte contenu dans le cartulaire de la cathédrale de Rouen ${ }^{34}$. Les Du

32. Magni Rotuli, t. I, p. 80 et t. II, p. 306

33. Cartulaire de Montebourg, BnF., ms lat. 10087, n 593, p. 180.

34. Trois chartes concernent la maison de Raoul Fils Étienne dans le cartulaire de la cathédrale de Rouen, Rouen, Bibl. mun., ms Y44: n 84, 176, 177, fol. 68v, 108v et 109. L'acte de Raoul, chancelier 


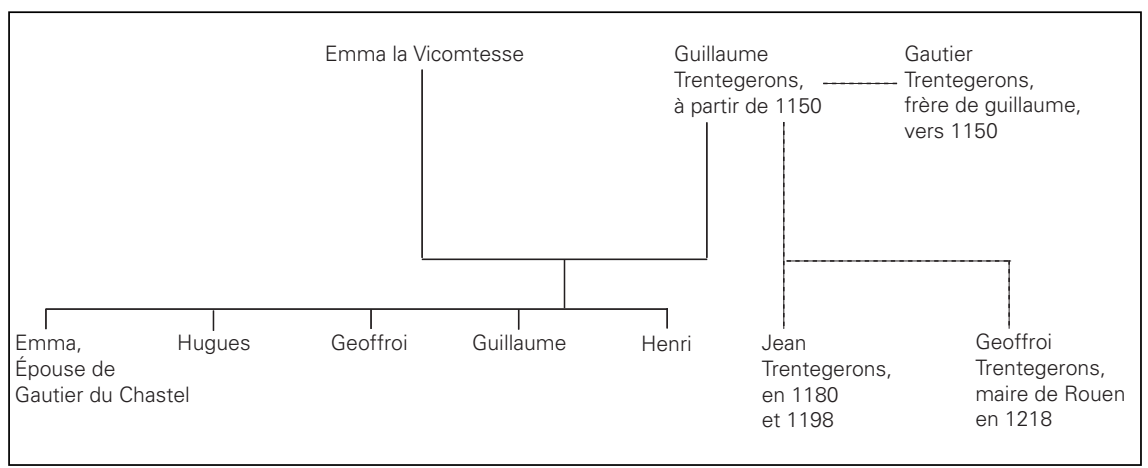

Le mariage d'Emma : première hypothèse.

Chastel constituaient une des grandes familles rouennaises, qui donna notamment un maire à la commune: Robert du Chastel, en 1181. La charte d'Emma fille de la Vicomtesse et de Gautier du Chastel est confirmée par une charte de Raoul [de Varneville], chancelier du roi Henri II de 1173 à 1182, associé à Guillaume de Malpalu, justicier du roi et à Barthélémy Bataille, maire de la commune de Rouen. D’après L. Delisle, Gautier de Coutances devait être à cette époque le lieutenant de Raoul de Varneville. Les témoins de cet acte furent Hubert chanoine de Lisieux, maître Thibaud, chanoine de Tours, Richard de Malpalu, maître Eudes de Coutances, Guillaume de la Mare et son frère Robert de la Mare, Gilbert Rainfroi, Gautier Fils Géroud, Nicolas Groignet, Hugues et Geoffroi fils de la Vicomtesse, Clarembaud le Roux... La maison avait appartenu à Raoul Fils Étienne, désigné par la mention «mariti sui» se rapportant à Emma: [...] Noverit universitas vestra quod Walterus de Castello et Emma, uxor ejus, filia Vicecomitissae, dotalitium ejusdem Emme, scilicet decem marcas auri, in domo que fuit Radulfi Filii Stephani, mariti sui [...] vendiderunt [... $]^{35}$. La mère et la fille portant le même prénom, on peut se demander si Raoul Fils Étienne fut le premier mari d'Emma (fille) ou celui de la Vicomtesse. On peut noter qu'à aucun moment Emma fille de la Vicomtesse n'est dite fille de Raoul Fils Étienne. La syntaxe de la phrase pourrait renvoyer ejusdem Emme à Vicecomitissae, mentionnée juste avant, ou bien à Emma, femme de Gautier du Chastel, seule Emma expressément dénommée dans la phrase ${ }^{36}$. La question reste ouverte.

On sait malheureusement peu de choses sur Raoul Fils Étienne: il est mentionné dans une donation faite par lui au prieuré Saint-Lô de Rouen, contenue dans une charte de confirmation de Henri II Plantagenêt, entre 1154 et 1189, concernant 60 sous donnés aux moines sur ses moulins de Carville, au nord-ouest de Rouen ${ }^{37}$,

de Henri II, est transcrit dans DELISLE et BERGER, Recueil..., Introduction, p. 101 en note. Gautier du Chastel apparaît sous la mention Walterus de Castello dans le cartulaire de la cathédrale de Rouen (fol. 68v), transcrit Walterus de Castellione, par L. Delisle : « du Chastel » semble la meilleure appellation.

35. Cartulaire de la cathédrale de Rouen, Rouen, Bibl. mun., ms Y 44, fol. 68v.

36. Pour la Vicomtesse, on lirait plus vraisemblablement ejusdem Vicecomitissae.

37. Carville, com. Darnétal, Seine-Maritime, ch.-l. de cant. 
appartenant à la banlieue rouennaise ${ }^{38}$. Il apparaît également dans une charte de Roscelin fils Cléramboud, «chambellan » de Henri II, vers 1150, par laquelle il fonde et dote l'église de Saint-Gilles du Mont-aux-Malades: Raoul Fils Étienne y est témoin aux côtés du gendre de Roscelin, Étienne Fils Theard, de Renaud de Saint-Philibert et de Gautier Marc d'Argent ${ }^{39}$. Il s'agirait de l'un des actes les plus anciens du prieuré du Mont-aux-Malades. Dans cet acte de donation, accompagné par la confirmation de l'archevêque de Rouen Hugues d'Amiens, Roscelin porte le titre de camerarius domini regis Angliae. Il aurait donc été membre de la curia regis. Nous ne connaissons pas sa carrière mais le nom de Gautier Marc d'Argent, témoin de l'acte, est ici bien intéressant ${ }^{40}$. Raoul Fils Étienne est lui-même l'auteur d'une charte en faveur du Mont-aux-Malades par laquelle il donne l'église Saint-Pierre de Carville et la chapelle Saint-Ouen de Longpaon ${ }^{41}$, en présence de Durand prêtre, Evrard de Calineio, Guillaume son neveu, Robert et son frère Vuimond ${ }^{42}$.

On aperçoit donc un personnage important, témoin d'actes de membres de la cour, proche de l'un des plus influents prêteurs de l'époque, possessionné à Carville et Longpaon (moulins, églises), et privilégiant pour son salut non des donations aux grands établissements religieux rouennais mais plutôt à Saint-Lô et au Mont-auxMalades. Ce choix s'effectue de façon assez logique pour ce membre issu d'un milieu social et économique urbain, en pleine ascension, non aristocratique. La complémentarité entre les établissements de Saint-Lô, du Mont-aux-Malades et de la Madeleine de Rouen, du point de vue des donations de bienfaiteurs, dont les mêmes noms reviennent régulièrement dans les chartes, est un fait avéré ${ }^{43}$. Et rappelons que Geoffroi fils de la Vicomtesse perpétue cette tradition bienfaitrice en accordant un don de 10 sous aux lépreux, revenu à prendre sur une terre tenue par Cléramboud le Roux ${ }^{44}$.

Mais la fille de la Vicomtesse ne détenait pas seule les droits sur la maison de Raoul Fils Étienne. Il est en effet à noter que Hawise, femme de Bernard Comin, céda aussi à Gautier de Coutances la part qu'elle avait sur la dite maison qui avait appartenu à son frère Raoul Fils Étienne ${ }^{45}$. Hawise adressa à la cathédrale de Rouen la donation de sa part de la demeure rouennaise qui fut celle de son frère et celle d'Ivo de Grand Pont, vers 1165-1184. Les témoins de cet acte furent Hugues de Cressy, R. abbé de Mortemer, Guillaume de Malpalu, Guillaume de Bréauté, Gautier Fils Géroud,

38. GlanVILlE, Léonce de, Histoire du prieuré de Saint-Lô de Rouen, Rouen, Cagniard, 1890-1891, t. 2, p. 294.

39. LANGLOIS, abbé Pierre-Laurent, Histoire du prieuré du Mont-aux-Malades-lès-Rouen, 1120-1820, Rouen, Fleury, 1851, p. 398.

40. Des clercs au service de la réforme: études et documents sur les chanoines réguliers de la province de Rouen, Mathieu ARnOuX (dir.), Brepols, Tunrhout, 2000, p. 63. L'acte de Roscelin camerarius est édité p. 330-331 (source, Arch. dép. Seine-Maritime, 23 HP1).

41. Longpaon : quartier du bourg de Darnétal.

42. LANGLOIS, Histoire du prieuré..., p. 405.

43. ARNOUX, Des clercs..., p. 146.

44. ARnOUX, Des clercs..., p. 147

45. Voir note $34,3^{\text {e }}$ charte concernant la maison de Raoul Fils Étienne. 
Nicolas Groignet, Lucas du Donjon, Guillaume Cavalier, Geoffroi du Val Richer (le Changeur), Barthélémy Bataille, Nicolas de Dieppe, Roger de Beaumont ${ }^{46}$.

L'époux de Hawise, Bernard Comin, lui, est mentionné dans l'Introduction au recueil des chartes de Henri II Plantagenêt de L. Delisle : il fut un témoin rouennais régulier des cas de justice du règne. Dans un acte de Henri II passé à Quevilly ${ }^{47}$ par lequel il donne aux lépreux (du Mont-aux-Malades) la foire de Saint-Gilles, qui durerait huit jours, ainsi que d'autres biens, apparaît, parmi les témoins, Bernard Comin, aux côtés de Guillaume comte de Mandeville, Hugues de Lacy, Séhier de Quincy, Jean de Soligny, Guillaume de Saint-Jean, Robert d'Estouteville et Jean Fils Luc, dont il est indiqué qu'il fut "depuis évêque d'Evreux» ${ }^{48}$. Bernard Comin, outre son lien avec la famille d'Emma la Vicomtesse, bénéficiait très certainement d'un accès à la cour du roi. D'après L. Delisle, une charte de l'abbaye du Bec constaterait sa donation de 100 acres de terre, qui lui avaient été données par l'impératrice Mathilde, dans le pays de Caux ${ }^{49}$. Il y est qualifié de senior. D'après le chanoine Porée, ce domaine était situé dans la portion défrichée de la forêt de Lillebonne, sur la paroisse de Saint-Martin de Gommerville ${ }^{50}$. Il était concédé à la condition que Bernard Comin et son fils Robert soient reçus religieux à l'abbaye du Bec, quand ils le voudraient. D'après une charte confirmative de Richard Cœur de Lion, Bernard Comin serait effectivement devenu moine au $\mathrm{Bec}^{51}$. Le roi Henri II en 1181 ou 1182 confirma la vente de terrains situés à Rouen, que Bernard Comin avait faite à Gautier de Coutances. Richard Cour de Lion confirma en 1190 l'acquisition du moulin de la Ruelle à Carville que l'abbesse de Saint-Amand avait fait de Bernard Comin, Hawise sa femme et Guillaume leur fils aîné ${ }^{52}$. On retrouve un sceau à son nom en 1226, à moins qu'il ne s'agisse de celui de son descendant, dans une nouvelle cession de ses droits sur les moulins de Carville, dans le fonds de l'archevêché de Rouen ${ }^{53}$. Il semblait donc posséder des droits sur ces moulins comme son beau-frère Raoul Fils Étienne.

La famille Comin semble par ailleurs bien présente à Rouen : sans liens de parenté prouvés, on constate la présence, vers 1206, d'un Richard Comin, à qui Hugues

46. Cartulaire de la cathédrale, Rouen, Bibl. mun., ms Y44, fol. 68.

47. Quevilly, Seine-Maritime, cant. Grand-Couronne.

48. LANGLOIS, Histoire du prieuré..., p. 399. Il s'agirait de Jean I Ir , évêque d'Evreux (1181-1192), originaire de Rouen, chanoine de la cathédrale entre 1156 et 1181 environ, clerc de Richard archevêque de Cantorbéry, de Gautier de Coutances archevêque de Rouen et du roi Henri II, et qui fut nommé en 1181 ou 1182 évêque d'Evreux. Un de ses parents, Luc, fut évêque à son tour entre 1203 et 1220 (Chassant, Alphonse, Histoire des évêques d'Evreux, Evreux, Tavernier, 1846, p. 51-52 et 57-59).

49. BnF, ms lat. 13905, fol. 26v et PORÉE, Chanoine Adolphe André, Histoire de l'abbaye du Bec, Evreux, Hérissey, 1901, t. I, p. 342.

50. Lillebonne, Seine-Maritime, ch.-1. de cant.; Gommerville, Seine-Maritime, cant. Saint-Romainde-Colbosc.

51. PORÉE, Histoire de..., t. I, p. 342, note 2.

52. LE CACHEUX, Marie-Josèphe, Histoire de l'abbaye de Saint-Amand de Rouen des origines à la fin du XVI ${ }^{e}$ siècle, Caen, 1937, p. 253-254.

53. Demay, Germain, Inventaire des sceaux de la Normandie, 1881, Paris, Imprimerie nationale, $\mathrm{n}^{\circ} 193$, p. 23. 
le Portier avait vendu ses manoirs de Perriers (-sur-Andelle), sans l'assentiment des moines de Saint-Ouen ${ }^{54}$. Guillaume Comin, entre 1171 et 1181 , fut témoin d'une concession de Richard, abbé de Saint-Ouen, à Robert frère du prêtre Géroud, ainsi que d'une vente par Durand et sa femme Ooldis à Richard de Malpalu, clerc de la cathédrale, d'un héritage rue Saint-Amand à Rouen ${ }^{55}$. Au XIII ${ }^{\mathrm{e}}$ siècle, le patronage de l'église Saint-Clément (près de la porte Saint-Clément, côté sud de l'enceinte de la cité, près du donjon de Rouen, auquel l'église fut longtemps étroitement liée) appartenait à une famille Comin, qui aurait été titulaire de plusieurs charges au palais dans les années 1160 et que les textes montrent implantée dès cette époque à Rouen ${ }^{56}$.

En tous les cas, la maison de Raoul Fils Étienne semble gérée après sa mort par deux femmes, Emma et Hawise, toujours mentionnées avec leurs maris. Si Emma (fille) se charge elle-même de la vente de ses droits sur la maison qui constituait vraisemblablement un douaire (dotalitium), dotation du mari en faveur de son épouse au moment du mariage, plusieurs hypothèses voient le jour. Soit Raoul Fils Étienne fut bien le mari d'Emma la Vicomtesse et on peut penser qu'à cette époque, vers 1182, la Vicomtesse avait disparu, car sinon elle aurait probablement figuré parmi les témoins de l'acte (les mentions la concernant encore dans le rôle de 1198 ne seraient alors que des rappels d'une activité passée); soit Raoul Fils Étienne fut le premier mari d'Emma fille, dont elle reçut la maison en douaire, ce qui expliquerait pourquoi la Vicomtesse n'intervient pas dans cette transaction.

Par ailleurs, la présence, parmi les témoins de l'acte, de Geoffroi et Hugues fils de la Vicomtesse, ainsi que d'un membre de l'Église de Lisieux (en lien avec Guillaume, fils de la Vicomtesse?) agit en faveur de l'appartenance à une même fratrie pour les enfants de la Vicomtesse. Il reste intéressant de noter, que contrairement à sa mère, Emma fille fut alors très entourée par les hommes de son entourage (mari, frères). Même si nous ne possédons pas d'acte similaire pour la Vicomtesse, qui apparaît principalement dans des comptes et des documents financiers, son cas gagne donc en mystère. Emma-fille n'hérita pas de l'apparente indépendance de sa mère. Mais il nous manque évidemment la connaissance exacte de la généalogie familiale et de la transmission des domaines familiaux, pour comprendre les droits des membres masculins et féminins de la famille, les modalités de l'exercice par les femmes de leurs droits en l'absence des hommes.

Emma la Vicomtesse a-t-elle été mariée avec le père de ses enfants? Les actes de donations concernant Raoul Fils Étienne ne mentionnent jamais d'épouse à ses côtés (à la même époque et dans un milieu social rouennais similaire, Bernard Comin associait fréquemment sa femme et son fils, par exemple). Pourtant, sa situation de veuve expliquerait plus aisément sa promotion sociale. Les premiers documents qui mentionnent Raoul Fils Étienne dateraient du tout début du règne de Henri II, à

54. Rouen, Arch. dép. Seine-Maritime, 14H 189. Perriers-sur-Andelle, Eure, cant. Fleury.

55. Rouen, Arch. dép. Seine-Maritime, 14H 714 et G 4263.

56. Delisle Et Berger, Recueil..., Introduction, p. 357 et 395. 


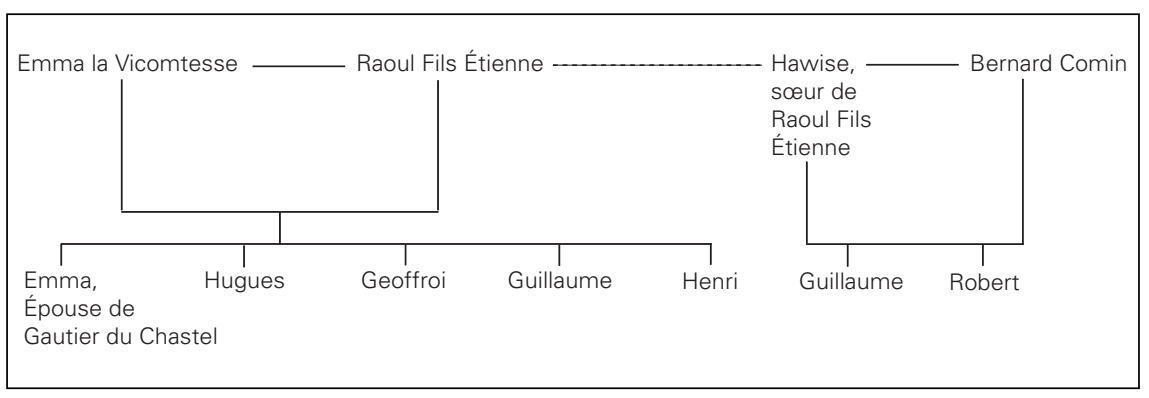

Le mariage d'Emma : seconde hypothèse.

une époque où Emma semble déjà exercer des fonctions importantes. Mise à part la quasi certitude qu'au moment de la cession des droits sur la maison du Grand Pont par la fille de la Vicomtesse, vers 1182, Raoul Fils Étienne avait disparu, on ignore la date de sa mort. Le début de la carrière de la Vicomtesse se situerait un peu avant 1158: soit Raoul Fils Étienne était décédé avant cette date et c'est une veuve qui devint Vicomtesse, soit Emma exerça ses activités du vivant de son mari... soit elle ne fut jamais mariée à Raoul Fils Étienne et les conditions de son ascension demeurent obscures. De plus, est-elle restée la veuve d'un seul homme ou connut-elle plusieurs veuvages successifs? Les lacunes du dossier incitent à rester prudent.

Un point reste important: nous n'avons pas retrouvé d'acte en son nom propre, ni de sceau, puisqu'Emma nous est principalement connue par les rôles de l'Échiquier. Seules ses apparitions dans des actes d'établissements religieux, par des mentions dans les cartulaires et obituaires (Saint-Victor de Paris, Saint-Lazare de Paris, Saint-Wandrille, cathédrale de Rouen), nous fournissent des renseignements supplémentaires sur ses activités propres. Dans le domaine de l'écrit, sa situation demeure ainsi proche de ses contemporaines, malgré une fonction traditionnellement dévolue à un homme. Cependant, puisqu'aucun mari ne lui est officiellement attribué et en vertu de ses responsabilités et de son autorité présumée, on s'attendrait à ce qu'elle fût plus fréquemment l'auteur d'actes. Mise à part sa participation à une possible compagnie marchande avec des hommes, elle semble agir pleinement seule, et non dans l'ombre d'un mari ou d'un tuteur en général. Le sceau le plus ancien retrouvé pour la vicomté de Rouen date de 1283 seulement ${ }^{57}$ : le fait qu'elle fût une femme n'expliquerait pas obligatoirement qu'elle en fût dépourvue. Il est possible que ses pairs masculins n'en aient pas possédé non plus à cette époque. En revanche, son fils Geoffroi en possédait un vers 1185, au moment de la donation d'une terre située à Franqueville. Répertorié dans l'inventaire de G. Demay, il représenterait

57. DEMAY, Inventaire..., n 1913, p. 201, sceau de la vicomté de Rouen dans une transaction au sujet du patronage de l'église Saint-Martin-le-Gaillard (Arch. dép. Seine-Maritime, fonds de l'archevêché de Rouen). 
un cavalier, tête nue, en costume civil, un faucon au poing: une iconographie singulièrement aristocratique ${ }^{58}$.

Dans la perspective de l'accès d'Emma à la fonction vicomtale, il est utile de revenir sur quelques précisions. Le titre de vicomte était donné aux membres de la bourgeoisie qui agissaient au nom d'une corporation ayant pris à ferme un domaine ou des revenus ducaux ${ }^{59}$. Il était à l'origine le chef officiel de la ville, issu du gouvernement ducal et, au milieu du XII ${ }^{\mathrm{e}}$ siècle, comme en Angleterre, il était généralement un membre d'une des familles dirigeantes de la ville. Le lien avec l'Angleterre était assez fréquent: le vicomte Anselme souscrivit par exemple plusieurs actes de Henri I ${ }^{\text {er }}$ Beauclerc dans les années 1130, en qualité de vicomte de Rouen, relatifs à la Normandie, mais aussi au Sussex et au Hampshire. Il n'était donc pas incroyable de tenir deux offices, en grande partie financiers, en Angleterre et en Normandie. Le passage de la Vicomtesse de Rouen à Southampton en découle assez naturellement. Il a été souvent constaté au XII ${ }^{\mathrm{e}}$ siècle une certaine perte de prestige de la fonction du vicomte de Rouen par apport au XI' siècle: elle tendit en effet à revêtir surtout un aspect financier, moins «politique ». Puis elle fut affermée, et ceci même à des bourgeois, et même à une femme, avant d'échoir à la commune elle-même. Pour exemple, la vicomté de Dieppe en 1180 était tenue par huit fermiers au total ${ }^{60}$. De plus, alors qu'en 1180, l'argent des fermes - comme celle de la vicomté de Rouen - constituait encore la plupart des revenus du duché, ce n'était déjà plus le cas en 1194$1195^{61}$. La valeur des fermes normandes baisse à la fin du XII ${ }^{e}$ siècle, témoignant d'une nouvelle diversité des sources de revenus: les fermes deviennent alors des sources secondaires et les responsabilités des «fermiers» ducaux sont par conséquent amoindries. Il ne s'agit bien entendu pas de dénigrer l'exploit dans la promotion sociale d'Emma, mais de replacer la fonction vicomtale dans son évolution chronologique, à un moment charnière de son existence puisque progressivement la commune de Rouen la rendit en quelque sorte obsolète.

\section{Le groupe social d'Emma la Vicomtesse}

Vers 1185, donc, Geoffroi, fils de la Vicomtesse, céda à Nicolas, fils de Luc du Donjon, son filleul, sa terre de Franqueville, tenue en fief de l'abbé de Saint-Ouen. Il était convenu que ledit Nicolas tiendrait la terre du donateur pour une livre de

58. Ibid., n² 2078 p. 222. Germain Demay indique que ce sceau était contenu dans le fonds de l'abbaye Saint-Ouen de Rouen (Arch. dép. Seine-Maritime) : il s'agirait de la donation passée en présence de l'abbé Samson vers 1185, qui n'est aujourd'hui munie que du sceau abbatial. Le sceau de Geoffroi a disparu.

59. Bouvris, Jean-Michel, "Contribution à une étude de l'institution vicomtale en Normandie au $\mathrm{XI}^{\mathrm{e}}$ siècle. L'exemple de la partie orientale du duché: les vicomtes de Rouen et de Fécamp », in Autour du pouvoir ducal normand $X^{e}-X I I^{e}$ siècles, Cahier des Annales de Normandie, $\mathrm{n}^{\circ}$ 17, Caen, 1985, p. 149-174.

60. Delisle ET Berger, Recueil..., Introduction, p. 213.

61. Moss, Vincent, «Reprises et innovations... », p. 94. 
poivre par an; il donna à celui-ci un besant d'or «de recognitione». L'accord fut passé en présence de l'abbé de Saint-Ouen Samson, dont l'acte est muni du sceau. Parmi les témoins figurent Jean Luce, Barthélémy Bataille, Nicolas Groignet, Geoffroi le Changeur, Silvestre le Changeur, Mathieu le Gros, Mathieu le Petit... ${ }^{62}$ Le paiement en poivre et le besant d'or indiquent clairement l'aisance de l'acheteur. Il suffit d'observer la liste des maires de Rouen depuis le milieu du XII ${ }^{e}$ siècle pour constater que parmi les témoins, plusieurs furent des personnages illustres de Rouen: Mathieu le Gros fut maire au moins en 1195, 1198, 1199, 1200; Jean Luce en 1206, 1210-1211, 1213-1217; Silvestre le Changeur en 1209; un Groignet (Raoul) fut maire en 1201 et 1203 . Cette charte a donc le mérite de nous présenter un accord concernant la cession d'une terre dépendant de l'abbaye de Saint-Ouen, principalement attestée par un personnel municipal en pleine ascension.

Un lien apparaît clairement entre la famille de la Vicomtesse et la famille du Donjon, grand lignage apparenté à l'émergence de la commune de Rouen. La famille des Du Donjon pourrait être liée à la Tour de Rouen, située au bord de la Seine, dans l'angle sud-ouest de la cité ${ }^{63}$. C'était la tour du palais des ducs de Normandie, construite entre 945 et 996 sous Richard ${ }^{\text {er }}$, et ce fut le premier donjon de pierre attesté en Normandie. Le lien établi traditionnellement entre l'église SaintPierre du Châtel, appuyée contre la muraille de la ville et proche d'une tour, et la fortification du donjon située dans l'angle sud-ouest de l'enceinte de la cité n'est pas évident: le donjon et le châtel correspondaient à deux lieux différents, qui ont donné l'un et l'autre leur nom à une famille rouennaise. Les Du Châtel étaient, on l'a vu, également liés à la famille de la Vicomtesse, par l'intermédiaire de sa fille Emma. Le site du donjon au XI ${ }^{\text {e }}$ siècle était occupé par un certain Rabel, premier membre connu du lignage des sires de Tancarville, que Guillaume de Jumièges présente comme le chef de la flotte du duc Robert le Magnifique (1027-1035), et dont on sait aussi que, par privilège spécial, il détenait des droits sur un grand nombre de ports de la basse vallée de la Seine et de la côte du pays de Caux ${ }^{64}$. L'appellation «du Donjon» étant avant tout liée à la fonction occupée par la famille à l'égard de ce bâtiment, il est probable que ce vocable ne fut pas exclusivement réservé à la ville de Rouen.

On pourrait peut-être rattacher les Du Donjon de Rouen de Rahier du Donjon, seigneur de Muzy (Eure, arrond. d'Evreux, cant. de Nonancourt), qui fonda en 1144 l'abbaye cistercienne de l'Estrée ${ }^{65}$. Astrid Lemoine, qui a étudié cette famille,

62. Rouen, Arch. dép. Seine-Maritime, 14 H 916.

63. Magni Rotuli, I, famille du Donjon, p. cxv. Voir SADOURnY, Alain, "Une famille rouennaise à la fin de la période ducale : les Du Donjon ", in La ville médiévale en deçà et au-delà de ses murs : Mélanges J-P. Leguay, Rouen, Publications de l'Université de Rouen (PUR 288), 2000, p. 183-188.

64. LE MAHO, Jacques, "Recherches sur les origines de quelques églises de Rouen ( $\mathrm{VI}^{\mathrm{e}}-\mathrm{XI}^{\mathrm{e}}$ siècles) », Bulletin de la commission départementale des Antiquités de la Seine-Maritime, t. 43, 1996, p. 193.

65. Le Prevost, Auguste, Mémoires et notes pour servir à l'histoire du département de l'Eure, recueillis et publiés par Léopold Delisle et Louis Passy, Evreux, Hérissey, 1864, vol. 2, p. 429 et LEMOINE, Astrid, La frontière normande de l'Avre de la fin du Xe siècle au début du XIII siècle: la défense et les 
suggère que le patronyme «du Donjon » fut peut-être celui qu'ils portaient avant de se retrouver à la tête de Muzy, ce qui tendrait à montrer que leur implantation dans ce fief fut tardive, au début du XII ${ }^{\mathrm{e}}$ siècle. D’autres «du Donjon » existent à cette époque, que ce soit dans l'entourage royal parisien, ou au sein d'autres familles normanno-chartraines: l'appellation paraît assez fréquente. Les prénoms de ces derniers (Henri, Anselme, Barthélémy, Ferry pour Paris; Baudry, Amaury, Pierre pour Chartres) ne rejoignent jamais ceux de Lucas, Laurent et Nicolas à Rouen. Ces familles pouvaient très bien devoir leur appellation à l'existence d'un donjon sur leur territoire d'origine et à la charge effectuée par leur ancêtre à l'égard du bâtiment. Pierre du Donjon, par exemple, bienfaiteur de la léproserie de Beaulieu à la fin du XII ${ }^{e}$ siècle, tirerait peut-être son nom de famille de la charge qu'il remplissait au château de Dreux ${ }^{66}$. Les connexions de ces Du Donjon avec la Normandie sont assez rares : Rahier du Donjon est témoin en 1155 d'une charte de Simon d'Anet, à la demande de son seigneur Galeran de Meulan, en faveur de la Trinité de Beaumontle-Roger ${ }^{67}$. Mais la fondation de l'Estrée est la seule occasion où il est directement lié à des Normands, puisqu'il agit en association avec deux habitants de Nonancourt, Amalric et Nivard, et avec Raoul d'Ilou et Hugues du Châtel ${ }^{68}$. Pour autant, aucun lien ne semble attesté à ce jour entre la famille de Rahier et les Du Donjon de Rouen.

Lucas du Donjon fut maire de Rouen en 1189 et 1194, tandis que Laurent et Nicolas du Donjon apparaissent dans les sources au tout début du XIII ${ }^{\mathrm{e}}$ siècle (Laurent était déjà mentionné dans le rôle de 1198, il serait mort avant 1248). Laurent apparaît comme témoin de la concession de Raoul de Cailly aux notables de la halle de Rouen d'une terre qu'il possédait à côté de celle-ci: ce local situé paroisse Saint-Eloi, près des quais de Seine, constituerait le premier hôtel de ville de Rouen ${ }^{69}$. Laurent du Donjon apparaît de nombreuses fois comme témoin, avant et après 1204, et semble par ailleurs très lié à Jean sans Terre ${ }^{70}$. Cependant, il ne devint pas maire : était-ce dû à un changement de personnel politique à Rouen après 1204 ? Laurent a pu jouer un rôle important auprès de Jean sans Terre dans les dernières années du règne normand, effectuant des achats pour le pouvoir royal, approvisionnant des garnisons en Normandie, prêtant parfois de l'argent au roi $^{71}$. En 1203, Jean sans Terre lui confie les revenus de la vente de charbon et de bois dans la ville de Rouen ${ }^{72}$.

structures de peuplement, Thèse de doctorat préparée sous la direction de François Neveux, Université de Caen, 2003, 3 vol., dactyl., vol. I, p. 45-57.

66. Jusselin, Maurice et MERLET, René, Cartulaire de la léproserie de Beaulieu, Chartres, Garnier, 1909, p. 69-70, n 172, 199.

67. Deville, Étienne, Cartulaire de l'église de la Sainte-Trinité de Beaumont-le-Roger, Paris, Champion, 1912, p. 31-32, n XXVII.

68. Rouen, Arch. dép. Eure, H 319, fol. 1-2, n ${ }^{\circ} 1$.

69. Rouen, Arch. mun., tiroir 417.

70. SADOURNY, Alain, «Une famille rouennaise...», p. 183-188.

71. Ibid.

72. Rotuli Chartarum in Turri Londinensi asservati accurante, éd. Thomas Duffus HARDY, Londres, 1837, Jean 5, p. 106. 
Qualifiés de "mercatores Rothomagenses", les Du Donjon auraient commercé activement avec l'Angleterre. Le rôle de Laurent est connu grâce aux sauf-conduits qui lui sont accordés par Jean sans Terre après 1204, afin qu'il puisse aller et venir en toute sécurité en Angleterre, avec ses marchandises, dont du vin ${ }^{73}$. En effet, la conquête de la Normandie par Philippe Auguste s'est traduite par la perte pour les Rouennais de tous les privilèges obtenus en matière de commerce: ils sont ainsi victimes de saisies et d'emprisonnements dans les ports anglais qu'ils fréquentent. Par la suite, Laurent du Donjon continue à bénéficier des mêmes avantages sous Henri III, tout comme son fils Nicolas.

Toujours parmi les témoins de la charte de Geoffroi fils de la Vicomtesse, se retrouve Mathieu le Gros qui rendait compte également pour la ferme de la vicomté de Rouen, avec Raoul Groignet et Raoul de Cailly, en $1198^{74}$. Il fut également associé à Geoffroi le Changeur, avec Raoul de Cailly, pour ces redditions de comptes ${ }^{75}$. Il assista à la rédaction des coutumes et ordonnances des moulins de Rouen en 1199, aux côtés de Guillaume Fils Raoul, sénéchal ${ }^{76}$. Il fut l'un des témoins du seul document légal juif en hébreu, rédigé du temps du maire Raoul de Cailly ${ }^{77}$. Il semble qu'il possédait à Rouen une parcelle dans le quartier juif, tout comme Jean de Saint-Candide ${ }^{78}$. Il fut témoin en tant que maire des donations de Renaud et Geoffroi du Bosc pour l'abbaye Saint-Ouen, aux côtés de Lucas Fils Jean ${ }^{79}$. Mathieu fit personnellement une donation à la cathédrale de Rouen, avec sa femme Mathilde, où l'on retrouve parmi les témoins les membres connus de la commune de Rouen: Clarembaud Le Roux, Nicolas de Dieppe, Jean Fessard, Raoul Groignet, Laurent du Donjon, aux côtés de chevaliers comme Henri de Hosa, Robert de Fresquiennes ${ }^{80} .$. Mathieu le Gros, maire, apparait dans une convention entre le prieur de Saint-Lô de Rouen et les religieux de Beaulieu au sujet de l'élection du prieur de Beaulieu, en $1200^{81}$. Fréquemment sollicité par Jean sans Terre pour des emprunts ou des réquisitions, il contribua à approvisionner plusieurs châteaux, celui de Verneuil tenu par Henri de Gray, celui de Montfort tenu par Jean de Préaux ${ }^{82}$. Il fut témoin de la capitulation de Rouen en $1204^{83}$.

73. Rotuli Litterarum Patentium in Turri Londinensi Asservati, éd. Thomas Duffus HARDY, Londres, 1835, I, p. 19b, 30 déc. 1205 et p. 86a, 10 sept. 1208.

74. Magni Rotuli, II, p. 6, 7, xx, xliv.

75. PACKARD, Sydney Raymond, Miscellaneous Records of the Norman Exchequer (1199-1204), Smith College Studies in History, vol. XII, n 1-4, oct. 1926 - juill. 1927, Nothampton, Mass, p. 73.

76. DeLISLE, Léopold, "Cartulaire normand de Philippe-Auguste, Louis VIII, Saint-Louis et Philippe le Hardi », in Mémoires de la Société des Antiquaires de Normandie, t. 16, Caen, 1852, p. 160-390, $\mathrm{n}^{\circ} 50$.

77. Golb, Norman, Les juifs de Rouen au Moyen Âge: portrait d'une culture oubliée, Rouen, Publications de l'Université de Rouen, 1985, p. 285-286.

78. Ibid., p. 278 et 281.

79. Rouen, Arch. Dép. Seine-Martime, 14 H 18, p. 487-489 et 531.

80. Rouen, Bibl. mun., ms Y44, n² 241.

81. Glanville, Histoire du prieuré..., t. 2, p. 297.

82. Rotuli Litterarum Patentium..., I, 30.

83. Magni Rotuli..., p. 6 et 7. 
Mathieu le Gros possédait un sceau pour ses actes à l'extrême fin du XII siècle, vraisemblablement en qualité de major de Rouen, mais cet avantage était également accordé à ses fils Amauri et Roger le Gros, co-donateurs avec leur père de rentes dans une charte du fonds du chapitre de Rouen ${ }^{84}$. Cet acte semble davantage être la donation privée d'une famille, qu'un acte officiel impliquant la commune de Rouen. Dans ces conditions, les autres notables de la ville, contemporains de Mathieu le Gros, ont pu posséder leur propre sceau, mais aucun ne nous est parvenu. Il reste intéressant qu'un membre de cette classe dirigeante émergente ait scellé ses actes individuellement ; cependant, il est vrai que l'usage du sceau s'était considérablement répandu au sein de la société médiévale et ne constituait plus la prérogative exclusive de l'aristocratie ou des établissements ecclésiastiques. Par ailleurs, le sceau d'un maire reste rare à cette époque en Normandie, d'autant plus le sceau d'un maire identifié individuellement. À la même période, en 1195, la ville de Meulan, possède un sceau représentant les têtes des 12 échevins superposées avec une fleur de lys, le major figurant seul au verso, non nommé, tenant une baguette, avec les mentions correspondantes «sigillum concionis de Meulent» et «sigillum majoris Mellenti ${ }^{85}$.

Raoul Groignet, lui, figure également dans la charte des ordonnances des moulins de Rouen de 1199, et il fut un des témoins de la loi juive rédigée à Rouen, avec le maire Raoul de Cailly, Geoffroi abbé de Saint-Ouen, Mathieu le Gros, Jean Luce, Étienne de Longchamp ${ }^{86}$. Raoul Groignet était fils de Benoît qui fut chanoine de la cathédrale, il occupa un poste important dans la gestion des finances en 1198, fut maire en 1201 et 1203, et l'un des représentants de la capitulation en 1204 avec Guillaume Groignet, fils de Nicolas Groignet. Nicolas Groignet fut témoin de la vente par Richard d'Illeville à Durand du Pin d'une terre dépendant de l'abbaye Saint-Ouen, à la fin du XII ${ }^{e}$ siècle, aux côtés de Geoffroi le Changeur, Barthélémy Bataille, Jean de Saint-Candide ${ }^{87}$.

Comme les Groignet, famille liée à la cathédrale de Rouen et à la bourgeoisie communale, il est sans doute possible de relier deux évêques d'Evreux à la famille bourgeoise des Luc, Luce ou Fils Luce ${ }^{88}$. Jean Luce fut maire de Rouen en 1206, 1210 1211 et 1213-1217. Les Fils Luce furent une famille à la destinée assez modeste, mais

84. DEMAY, Inventaire..., 1881, Paris, $\mathrm{n}^{\circ} 1711$, p. 175 : dans une donation de rentes sur des tènements dans les paroisses de Sainte-Marie-la-Petite et Saint-Patrice de Rouen (fonds du chapitre de Rouen), sceau rond, intaille représentant une tête de Minerve, au casque surmonté d'une aigrette et finissant par derrière en une tête «silénique»; l'inscription est "sigillum Mathei le Gros » mais ne mentionne pas sa fonction de maire de Rouen. $\mathrm{N}^{\circ} 1710$, p. 174 : sceau rond, intaille représentant Julie et Caracalla, bustes en vis-à-vis, "sigillum Amaurici et Rogeri, fratris suis». L'empreint à la mythologie et à l'histoire antiques et guerrières pour ces bourgeois de Rouen est particulièrement intéressante.

85. Ibid., n 1645 p. 168, sceau rond, Musée des Antiquités de Rouen.

86. Rouen, Arch. dép. Seine-Maritime, 14H 448 et GolB, Les Juifs de Rouen..., p. 285-286.

87. Rouen, Arch. dép. Seine-Maritime, 14H 796. Magni Rotuli..., éd. Lechaudé-d'Anisy et Charma, Nicolas Groignet, p. 7 et Raoul Groignet, p. 6 et 7.

88. Cf. supra, note 46 . 
que l'on retrouve également à Caen. Celle-ci semble descendre d'un ministerialis, exerçant une activité professionnelle spécialisée : l'exploitation, pour l'abbaye de la Trinité de Caen, des moulins du Petit-Odon, sources de revenus importants. Lucien Musset classait le lignage dans la catégorie « aristocratie de quartier »; il est certain que plusieurs membres étaient intimement mêlés aux maniements de fonds royaux ${ }^{89}$. Jean Fils Luce serait apparu dès 1171. La confusion est difficile à éviter entre Jean Fils Luce et Luc Fils Jean, Lucas filius Johannis, que l'on retrouve fréquemment dans les rôles de l'Échiquier normand ${ }^{90}$. Appartenaient-ils bien à la même famille ? Luc Fils Jean rendait compte à la toute fin du XII e siècle, vers 1198 , pour le bailliage du Roumois et pour la ferme de la vicomté de Rouen ${ }^{91}$. On le voit rendre compte de plus de 1054 livres du tallage prélevé dans le bailliage du Roumois, pour les garnisons $^{92}$. Selon un compte de Garin de Glapion, sénéchal de Normandie, dans le rôle de juin 1200-nov. 1201, 300 livres furent reçues du maire de Rouen des mains de Guillaume Belet et Guillaume Fils Luce ${ }^{93}$. Luc Fils Jean rendit compte également de la vente des vins de Philippe de Colombières et il était impliqué dans le transport des vins du roi de Harfleur à "l'île d'Andely " ${ }^{94}$. Il avait dû recevoir des biens en Angleterre car, après 1204, ses manoirs de Teignweek, Woburneford (Devon) et Wallington (Surrey) passèrent dans les mains du roi, jusqu'à ce que Eustachia sa femme offrît au roi 15 marcs pour obtenir la terre qui appartenait à son mari en Angleterre.

Geoffroi le Changeur, grande figure rouennaise, apparaît fréquemment dans les rôles de l'Échiquier ${ }^{95}$. En 1195, le compte de Geoffroi le Changeur concernant les sommes qui lui furent apportées pour la rançon du roi, formaient un total de plus de 27891 livres. D'après le rôle de 1195, Geoffroi le Changeur rendit compte des revenus des peaux, laine et sel à Rouen depuis la fête Saint-Nicolas jusqu'à la Pentecôte, pour une somme s'élevant à plus de 632 livres. Une partie de cette somme, 100 livres, était donnée au maire de Rouen pour les travaux dans la cité ${ }^{96}$. Geoffroi rendit aussi compte de 3000 livres reçues du Trésor de Caen. Une remise de 1000 livres venant de ce Trésor fut payée par Geoffroi au Chambellan. Henri de Gray, fermier de la praepositura de Verneuil, rendit compte, toujours en 1195, de plus de 2156 livres, reçus partiellement du Trésor de Caen et du sénéchal de Normandie, par l'intermédiaire de Geoffroi le Changeur ${ }^{97}$. Il fut fréquemment associé aux personnages importants de la commune de Rouen: Roger Baudry, Raoul de Cailly,

89. MussEt, Lucien, «Essai sur la bourgeoisie caennaise (1150-1250)", in Recueil d'études offert au doyen Michel de Boüard, Annales de Normandie, n spécial, Caen, 1982, vol. II, p. 409-436.

90. PACKARD, Miscellaneous Records..., memb. 18; Magni Rotuli, II, 489 et p. 7, 49, 50, 51, 64, 76, 79, 100.

91. Ibid., II, p. cxi, cxiii, rôle de 1198 et p. 50.

92. Ibid., II, p. cxxv.

93. Ibid., II, p. ccxviii.

94. Ibid., II, p. ccxiv.

95. Ibid., I, p. cxl-cxli.

96. Ibid., I, p. clxv.

97. Ibid., I, p. clxvii. 
Jean Fessart, Nicolas de la Londe, Raoul de Cotevrard, Mathieu le Gros ${ }^{98}$... Dans le rôle de 1198, Geoffroi de Valricher, le Changeur de Rouen, rendit compte des dépenses faites par le roi Richard pour la défense de la frontière orientale de la Normandie ${ }^{99}$. Un autre compte rendu par Geoffroi concernait 1019 livres du tallage du bailliage de Caux, et le tout correspondait à une somme finale de plus 5514 livres angevines et de plus de 6859 marcs sterling, soit plus de 18291 livres angevines. De cette somme, environ 6500 marcs furent versés à Richard Brieguerre, maire de Rouen, qui rendait compte des revenus de la vallée de la Seine en 1198, depuis le pont de Rouen jusqu'aux Moulineaux ${ }^{100}$. À d'autres reprises, il est distinctement qualifié de membre de la famille des Valricher ${ }^{101}$. Henri de Pont-Audemer, bailli de Caux en 1198, rendit compte de l'emprunt pris dans le bailliage de Caux, soit 2050 livres, dont 1647 livres furent données à Geoffroi le Changeur, en raison des convois de vins du roi depuis Leure jusqu'à Rouen et aux châteaux ducaux ${ }^{102}$.

Parallèlement, l'essartement de la forêt de Lillebonne, attesté en 1180, fut poursuivi en 1195, sous la responsabilité de Silvestre le Changeur qui rendit compte de plus de 1607 livres de la vente de la forêt. Le prénom Silvestre était particulièrement rare à cette époque ; pourtant, on en trouvait un dans une autre grande famille de manieurs de fonds en Normandie à cette époque : Silvestre de Foro ou du Marché, dans une charte duquel on a vu que Geoffroi fils de la Vicomtesse figurait parmi les témoins ${ }^{103}$. Avec Gautier Marc d'Argent, membre d'un autre lignage très important, Silvestre le Changeur envoya de l'avoine à Bordeaux pour l'usage du roi ${ }^{104}$. Il fut maire de Rouen en 1209. Roland le Changeur, lui, aurait été également maire de Rouen, mais à une date que l'on ignore. Dans le grand rôle de 1198, aux côtés d'Emma la Vicomtesse, il répondit solidairement de 40 marcs, dette de Gervais de Hampton, avec deux autres inconnus.

\section{Un milieu rouennais}

L'influence de ces hommes avant l'accès officiel aux responsabilités communales ne fait aucun doute. Les rois surent d'ailleurs trouver dans l'octroi de concessions et d'établissements de gouvernements municipaux un moyen de tenir les villes en main : les Établissements de Rouen, initiés par Henri II, confirmés par ses successeurs, mirent peu à peu en place un système de gouvernement municipal au final assez contraignant et peu démocratique vers 1180, le roi conservant de fait une part importante du pouvoir et surtout des richesses produites par l'activité marchande

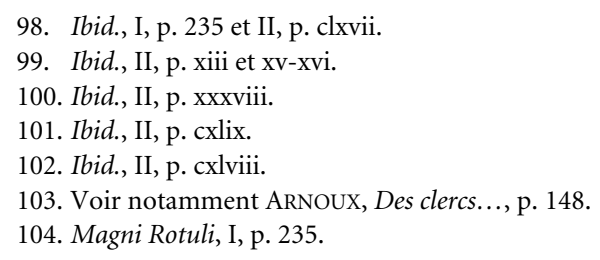


de la ville ${ }^{105}$. Mais l'existence des échevins précédait parfois de longtemps la rédaction d'une charte de commune. Les plus anciens privilèges concédés à l'ensemble

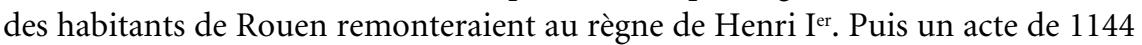
énumère les libertés des personnes et de leurs biens, des privilèges de juridiction, des dispositions fiscales et des privilèges commerciaux. Mais il n'y est pas décrit d'organisation communale. Dans une charte mentionnant explicitement la ghilda mercatorum, Henri II confirme et augmente ces privilèges vers 1150, puis en $1174^{106}$. Les Rouennais jouissent alors d'avantages à Londres et en Angleterre, du monopole du commerce sur la Basse-Seine et ils s'assurent la quasi-totalité des exportations de vins de France vers l'Angleterre. Les Établissements de Rouen à proprement parler doivent dater de 1180 environ. Ils sont confirmés en 1199 par Jean sans Terre puis par Philippe Auguste, après la prise de la ville en $1204^{107}$.

La présence de beaucoup de ces personnages dans les actes de la cathédrale de Rouen et des autres établissements religieux rouennais nous renseigne sur leur participation indéniable à la commune de Rouen. La bourgeoisie marchande de la ville était vraisemblablement représentée par 100 pairs, principaux bourgeois, qui choisissaient chaque année parmi eux le maire, 12 échevins et 12 conseillers pour le gouvernement de la commune ${ }^{108}$. Il est assez rare de découvrir une charte attestée par 24 personnes, en plus du maire, nous permettant d'observer le personnel communal en activité à un moment donné: la moyenne se situe davantage autour d'une dizaine de témoins. Cependant, Barthélémy Fergant, en 1177, figure dans le cartulaire de la cathédrale de Rouen, entouré de Guillaume Malpalu, Guillaume de Bréauté, Gautier fils de Géroud, Nicolas Groignet, Guillaume Cavalier, Roger Dorgueil, Luc du Donjon, Guillaume Petit, Geoffroi du Val-Richer, Hugues le Tuilier, Barthélémy Bataille, Richard Fils Benoît, Roger Baudri, Jean de Saint-Candide, Nicolas de Dieppe, Roger Fils de Guy, Roger de Beaumont, Raoul de Cotevrard, Raoul de Canville, Simon Naguet, Jordan Joismare, Raoul de la Vigne, Mauger de Saint-Lô, Guillaume et Robert de la Mare, Hugues et Geoffroi fils de la Vicomtesse, Clarembaud le Roux ${ }^{109}$. Vers 1180, une vente par Durand et Ooldis sa femme, à Richard de Malpalu, clerc de la cathédrale, d'un héritage rue Saint-Amand, dépendant du fief de Robert, fils de Clarembaud, à tenir de celui-ci par 36 sous-monnaie de Rouen de rente annuelle, fut attestée par Barthélémy Fergant, maire de Rouen, Guillaume de Malpalu, Barthélémy Bataille, Nicolas Groignet, Roger de Beaumont, Richard de Carville, Roger Dorgueil, Raoul de la Vigne, Simon Naguet ${ }^{110} \ldots$ Au tout début du XIII ${ }^{e}$ siècle, Jean

105. Delisle et BERGER, Recueil..., t. I, charte ${ }^{\circ}$ XIV et XV*, p. 18 et t. II, nº DXXVI, p. 89-90.

106. CHÉRUEL, Adolphe, Histoire de Rouen pendant l'époque communale, 1150-1382, Rouen, Périaux, 1843, t. I, p. 242.

107. Delaborde, Henri-François, Petit-Dutaillis, Charles et Monicat, Jacques, Recueil des actes de Philippe Auguste, roi de France, $1^{\text {er }}$ nov. 1194-31 oct. 1206, Paris, Imprimerie nationale, 1916 et 1943, t. II, n ${ }^{\circ} 789$, p. 362-367.

108. ChÉruel, Histoire de Rouen..., t. I, p. 51.

109. Rouen, Bibl. mun., ms Y44, fol. 60, fol. 69, fol. 107, 108v-109.

110. Rouen, Arch. dép. Seine-Maritime, G 4263. 
Luce, maire, Jean Fessart, Nicolas de Dieppe, Raoul le Juif furent ensemble témoins d'une concession faite à un chanoine de la cathédrale ${ }^{111}$; Clarembaud le Roux fut témoin de diverses donations faites par Gautier Roscelin au prieuré de Saint-Lô, qui souhaitait y être reçu religieux, aux côtés de Nicolas de Dieppe, de Philippe le Changeur, du temps du maire Robert du Chastel (vers 1181 ou vers 1220) ${ }^{112}$. Sans doute issu de la même famille, Étienne Clarembaud donna au prieuré Saint-Lô de Rouen 10 sous de rente, en même temps que le médecin Simon qui donna une maison située rue Saint-Nicolas ${ }^{113} \ldots$

On découvre ainsi tout un cercle de personnages dont les souscriptions se recroisent dans les chartes rouennaises de cette époque. Les Groignet, les Du Chastel, qui furent alliés à Emma la Vicomtesse, appartenaient aux lignages seigneuriaux les plus en vue dans la cité à partir de la seconde moitié du XII ${ }^{\mathrm{e}}$ siècle, et plusieurs de leurs membres furent maires de Rouen, même au XIII' siècle. On peut citer les Luce, les Fessart, les Fergant, les Le Gros, les Du Donjon, les Le Changeur-Val Richer, les de la Rive, les Marc d'Argent... Leur histoire s'étend parfois sur quatre ou cinq générations. Les stratégies familiales conduisaient aussi à investir les milieux ecclésiastiques, parallèlement au service de la commune. Certaines familles ont fourni, comme les Le Changeur et les Groignet, des membres au chapitre cathédral. Cependant plusieurs de ces familles disparurent assez rapidement, ce fut le cas par exemple des Groignet, des Du Donjon, des descendants d'Emma la Vicomtesse, dont on ne trouve plus trace, très rapidement, au-delà du début du XIII ${ }^{\mathrm{e}}$ siècle.

Jean sans Terre puisa volontiers dans les trésors des Rouennais. Il emprunta plusieurs fois des sommes considérables à Mathieu le Gros, maire de Rouen, soumettant la ville à des emprunts forcés. Les rôles de l'Échiquier normand conservés montrent une large part de la ferme impayée, jusqu'à 1198, quand un effort financier important fut demandé durant la guerre contre Philippe Auguste. La ville paya alors non seulement la ferme de 3200 livres angevines mais consentit aussi à des tallages et d'autres paiements, au total pour 7000 livres. Au XII et au début du XIII ${ }^{\mathrm{e}}$ siècle, plusieurs riches citoyens ont pu être largement impliqués dans les transactions financières avec le gouvernement normand et plus tard anglo-normand ${ }^{114}$. Mais Jean aliéna également ses droits sur Rouen : il confia le monopole du charbon et du bois qui se vendaient dans la ville à Laurent du Donjon; l'impôt prélevé sur chaque étal du marché à un des pairs de la ville, Geoffroi le Chambellan. Chacun d'eux devait au duc une paire d'éperons dorés, mais c'était là un simple hommage féodal ${ }^{115}$. Jean sans Terre désirait se concilier les bourgeois ou avait de l'intérêt pour

111. Ibid., G 4264 .

112. GLANVILLE, Histoire du prieuré..., t. 2, p. 326, pièce XVIII.

113. Ibid., t. 2 ; p. 376, pièce LV.

114. MUSSET, Lucien, «Y eut-il une aristocratie d'affaires commune aux grandes villes de Normandie et d'Angleterre entre 1066 et 1204 ?», in Études normandes, n 3, 1986, p. 7-19; Magni Rotuli, I, 70-71; II, 154-155, 304-305, 306-308.

115. Rotuli Chartarum..., p. 21 et 106. 
le commerce : il enjoignit par plusieurs chartes aux gardes des ports de ne pas molester les marchands de Rouen et de ne lever sur eux aucun impôt exorbitant. Il accordait parfois même des patentes à de riches bourgeois, pour faire du commerce avec l'Angleterre. On l'a vu, Laurent du Donjon obtint des sauf-conduits pour envoyer des vaisseaux dans les ports d'Angleterre ${ }^{116}$. Jean sans Terre ne dut se dépouiller de ces droits avantageux qu'en échange de quelque impôt considérable levé sur les bourgeois. En 1204, les nobles et les bourgeois stipulèrent avec Philippe Auguste une trêve, qui fut conclue par les chevaliers et par un certain nombre de personnages déjà rencontrés ${ }^{117}$. Parmi les notables rouennais, certains, comme les Valricher, survécurent au possible changement de personnel politique, même s'ils furent nombreux à avoir soutenu financièrement le gouvernement de Jean sans Terre.

\section{Conclusion}

L'attention portée à Emma et sa famille nous permet d'apercevoir simultanément certains de ces hommes nouveaux, d'origine non seigneuriale, comme ses propres fils, régulièrement présents comme témoins de donations et de ventes à Rouen. Il s'agit d'un groupe social nouveau, dont la fortune proviendrait du négoce, mais aussi de l'exploitation des revenus ducaux. Dans le cas de la Vicomtesse Emma, on voit comment la symbiose durant un siècle et demi de la Normandie et de l'Angleterre, permit le développement d'un milieu d'affaires commun aux deux rives de la Manche.

L'implication dans les mouvements de fonds du trésor royal a dû jouer un rôle non négligeable. Gilbert Becket, père de Thomas, membre d'une famille bourgeoise rouennaise, marié à une Caennaise, qui se fixa à Londres entre 1106 et 1119, en est un des premiers exemples significatifs : commerçant, il fut un temps sheriff de Londres. Si les affaires d'argent et la participation à l'administration royale paraissent avoir été plus importantes que le commerce pour la constitution de grandes fortunes, il est nécessaire de rappeler la nature de nos sources: les rôles de l'Échiquier, qui sont avant tout des documents financiers, tiennent entre 1180 et 1204 une place très importante, tandis que les documents économiques font à peu près défaut, ce qui peut fausser quelque peu notre point de vue, en ne nous faisant pas apprécier à sa juste valeur l'importance des activités commerciales. Un des grands moteurs de l'accession aux premiers rangs de la société avant 1204 paraît ainsi avoir été le

116. Rotuli Litterarum Patentium..., fol. 105.

117. Pierre de Préaux, Geoffroi du Bosc, Henri d'Estouteville, Robert d'Esneval, Thomas de Pavilly, Pierre de Hotot; les bourgeois étaient représentés par Robert de Malpalu alors maire de la ville, Geoffroi le Chambellan, Mathieu le Gros, Hugues fils de la Vicomtesse, Jean Luce, Raoul Groignet, Enard de la Rive, Jean Fessard, Clarembaud (le Roux), Jean Batiécoc, Roger Malasnon, Walon de la Rive, Osmond Poirier, Bernier le Fèvre, Guillaume Groignet, Guillaume Freschet, Robert de Mesnil-le-Lac, Auger de Dessus-la-Rive, Robert du Châtel, Nicolas de Dieppe, Robert Poirier, Robert Vilain, Roger Gautier, Sylvestre de Watteville, Martin de la Corveserie, Hugues, neveu de Walon, Richard de Saint-Wandrille, Geoffroi Vilain, Pierre le Pêcheur, Lucas Baudry, Guillaume le Meunier. 
maniement des fonds royaux, le change ou le prêt, quitte, sans doute, à faire ensuite fructifier l'argent ainsi acquis par le placement foncier et le commerce guère cité dans nos textes. Le cas d'Emma la Vicomtesse a le mérite de nous renseigner sur la vie d'une femme non issue de l'aristocratie, au XII siècle, en dehors des chartes de donations ou de transferts de biens immobiliers et en dehors des sources narratives, à travers lesquelles il est traditionnel d'étudier les femmes de la noblesse anglo-normande.

Emma la Vicomtesse, qui a pu prêter assez tôt des sommes importantes au pouvoir royal, dilapida impunément les revenus des fermes de plusieurs vicomtés, mais elle investit dans un terrain à Saint-Wandrille, tandis que sa famille semblait posséder des biens fonciers à Rouen et dans sa banlieue, et elle pratiqua le commerce des harengs et du sel sur la Seine... Il était stratégiquement nécessaire d'avoir plusieurs cordes à son arc. Prise à ferme des revenus ducaux et commerce: les clés de la fortune, dont les conséquences se retrouvent assez généralement dans l'investissement immobilier, en territoire urbain, et en rentes foncières, tout comme dans la constitution du patriciat urbain.

En près de 40 ans de présence dans les sources rouennaises (1158-1198 environ), l'entourage d'Emma s'est révélé des plus diversifiés. Le débat sur le rôle des femmes médiévales s'enrichit donc, dépassant leur statut de victimes de la domination masculine, et remettant sans cesse en cause l'existence de deux mondes séparés. On peut recenser ses relations purement marchandes avec des hommes tels que les Le Changeur; ses contacts avec les membres de la cour ducale, qui se portèrent garants pour elle, avec le roi lui-même et les officiers royaux; avec des abbayes telles que SaintWandrille, Saint-Victor ou Saint-Lazare de Paris - fondées au début du XII siècle -; avec le milieu ecclésiastique plus généralement, de par sa présence dans des nécrologes et à travers la vocation de son fils Guillaume. Cependant, elle ne semble pas avoir été une bienfaitrice régulière d'un établissement particulier. Ses choix ne semblent pas s'être portés, comme un certain nombre de ses contemporains, appartenant à la bourgeoisie rouennaise, sur le Mont-aux-Malades, Saint-Lô ou la Madeleine de Rouen. Elle est liée à de très grands établissements (cathédrale de Rouen, abbaye de Saint-Wandrille) : à travers ses donations, qui tendent davantage à imiter l'aristocratie de l'époque, c'est son statut social qui est encore mal délimité. Ses relations avec les établissements du Bassin Parisien sont plus explicites: Saint-Victor ou SaintLazare de Paris sont alors de fondation récente. Les actes dans lesquels apparaissent ses fils marquent plus clairement une distinction bourgeoise pour des établissements religieux urbains, de fondation également plus récente que les grandes abbayes prestigieuses du haut Moyen Âge.

On serait tenté de dire qu'Emma se situe encore à un moment charnière de l'évolution de la société médiévale du XII ${ }^{e}$ siècle, où la classe marchande urbaine cherche ses marques. Mais c'est sans compter les relations certaines de la Vicomtesse avec des membres de la haute aristocratie, voire avec le roi lui-même, qui ont pu l'influencer et lui faire adopter des modes d'action dignes de la noblesse anglo-normande. La mobilité sociale de l'époque nous est encore bien étrangère. Par ailleurs, l'étendue géographique de ses activités constitue une piste de recherche attirante. À l'exté- 
rieur de la région rouennaise, Emma est également liée à l'évêché de Lisieux et à des abbayes parisiennes. C'est surtout ses relations avec ces dernières qu'il conviendrait d'explorer de façon plus approfondie, car la perspective d'une activité commerciale entre Rouen et la région parisienne est peut-être l'une des clés de l'histoire de la Vicomtesse, qui avait déjà à son actif une expérience des affaires anglaises...

Enfin, Emma était très proche de ce personnel communal naissant, qui souscrivit des chartes la concernant et dont ses propres fils furent très certainement des membres importants. C'est l'origine des familles, qui dirigeaient la ville à cette époque, qui est ainsi mise en lumière: Emma ne possédait pas de nom faisant référence à un toponyme, ni à une lignée noble bien connue. Sa seule fonction la désignait, comme beaucoup d'autres bourgeois marchands, signe d'une évolution sociale économique et urbaine dont il reste encore beaucoup à découvrir.

\section{Récapitulatif :}

\section{les mentions concernant Emma ou ses enfants dans la documentation}

\section{Sources}

The Great Rolls of the Pipe for the [5th-34th] years of the reign of King Henry the second, ed. by The Pipe Rolls Society, Londres, 1884-1925: Pipe V, p. 50-51; Pipe VI, p. 22; Pipe VII, p. 58; Pipe VIII, p. 39; Pipe IX, p. 56; Pipe XII, p. 45.

Magni Rotuli Scaccarii Normannie sub Regibus Angliae, éd. Thomas Stapleton, Londres, Soc. Ant. Londinensis, 1840-1844, tome I, p. cvi, 78, 80, 284 et tome II, p. xxii, 305-306, 395.

Cartulaire de Saint-Wandrille, Arch. dép. Seine-Maritime, 16H, fol. 117v, n XV (acte de l'abbé Anfredus, vers 1165-1178).

Cartulaire de Saint-Lazare de Paris (1124-1254), Arch. nat., MM 210, fol. 41v.

Obituaire de la cathédrale de Rouen (XIII' ${ }^{e}$ siècle), Arch. dép. Seine-Maritime, G 2094, 2 septembre.

Obituaire de Saint-Victor de Paris, éd. Auguste Molinier, Recueil des Historiens de la France, Obituaires de la province de Sens, t. I. Diocèse de Paris et de Sens, Paris, 1902, p. 555, 24 avril.

Obituaire de Saint-Ouen de Rouen, Bibl. mun. Rouen, Missale Rothomagensis (XIII ${ }^{e}$ siècle) : ms A459 (276), fol. 4.

Cartulaire de Montebourg, BnF, ms lat. 10087, n593, p. 180.

Cartulaire de la cathédrale de Rouen, Rouen, Bibl. mun., ms Y44: n 84, 176, 177, fol. $68 \mathrm{v}, 108 \mathrm{v}$ et 109.

Fonds de Saint-Ouen de Rouen, Rouen, Arch. dép. Seine-Maritime, 14 H 916.

\section{Bibliographie}

Delisle, L. et Berger, E., Recueil des actes de Henri II Plantagenêt, Introduction, Recueil des Historiens de la France, Chartes et Diplômes, 1909-1927, p. 214218, p. 364 et p. 101 en note. 


\section{Vers 1181-1190}

Donation par Geoffroi, fils de la Vicomtesse Emma, en présence de l'abbé de SaintOuen Samson, à Nicolas, fils de Luc du Donjon, son filleul, de sa terre de Franqueville, que tiennent Richard Bidart pour 5 mines d'avoine et 5 chapons, Benoit du Moncel pour 3 mines d'avoine et 3 chapons, Raoul Lecomte pour 3 mines et un chapon, Osbert du Monstier pour 12 deniers et 3 chapons, Hugues de Poses pour une mine d'avoine et 2 chapons: le dit Nicolas tiendra la terre du donateur pour une livre de poivre par an; il donne à celui-ci un besant d'or «de recognitione».

Original, Archives Départementales de la Seine-Maritime, 14H 916. Parchemin. Sans date.

Sceau ogival, 70 millimètres (répertorié par G. Demay, Inventaire des sceaux de la Normandie, Paris, 1881, n² 2852, p. 318) : abbé debout, tête nue, crossé, tenant un livre.

«+ Sigillum Samsonis, abbatis Sancti Audoeni Rothomagensis »

Sciant omnes presentes et futuri quod ego Gaufridus filius vicecomitisse dedi et concessi et pre/senta carta confirmavi Nicholo filiolo meo filio Luce de Donion terram meam de/Frankevilla quam Ricardus Bidart tenebat de me unde annuatim reddebat $\mathrm{v}$ minas avene/ad festum beati Michaeli et $\mathrm{v}$ capones ad Natalem, Benedictus de Moncello iii minas avene ad festum/sancti Michaeli iii capones ad Natalem, Radulfus comes iii minas avene ad festum beati Michaeli et i caponem ad/Natalem, Osbertus de Monasteri xii denarios ad Natalem et iii capones, Hugo de Poses i minam avene ad/festum sancti Michaeli et ii capones ad Natalem, Rogerus molendini debet hunc redditum submonete/per unam piecam terre quam de eodo feodo tenet et unam piecam terre iuxta maram in dominico hanc terram/debet Robertus de Sancto Eligo adgitare usus abbatem Sancti Audoeni et alios dominos feodum per masagium quod ibi tenet/Hunc redditum concessi predicto Nicholo tenendum ei et heredibus suis de me et heredibus meis per unam/libram principis annuatim reddendam ad Natalem. Per hui autem donatoris concessiones dedit mihi predictus/Nicholaus i bisantius auri de recognitione et ego ei et heredibus suis debeo illum redditum garantirare per/predictum censum. Actum fuit hoc coram Sansone tunc abbate Sancti Audoeni et ei domine conventum unde/hoc feodum movet. Testibus his Johanne Luce, Roberto Belfiz., Bartholomeo Batalle, Nicholaus Grognet, Gaufrido Cambitor, Silvestro Cambitor,/ Matheo Grosso, Matheo Parvo, Reginaldo Clerico, Ricardo Filio Matheo et aliis multis. 